\title{
Impregnated central venous catheters should be readily used to reduce risk of bloodstream infection
}

\author{
Ruth Gilbert professor of clinical epidemiology ${ }^{1}$, Katie Harron research assistant ${ }^{1}$, Quen Mok \\ consultant intensivist ${ }^{2}$, Carrol Gamble professor of medical statistics ${ }^{3}$ \\ ${ }^{1}$ UCL Institute of Child Health, London WC1N 1EH, UK; '2Department of Paediatric Intensive Care, Great Ormond Street Hospital, London, UK; \\ ${ }^{3}$ MCRN Clinical Trials Unit University of Liverpool, Liverpool, UK
}

Smith and Nolan's overview of central venous catheters (CVCs) states that evidence from 56 trials shows that antiseptic or antimicrobial impregnation of CVCs reduces the risk of bloodstream infection related to their use. ${ }^{12}$ The dilemma for clinicians, however, is which types of impregnation work best and for whom.

The best available evidence on the type of impregnation comes from a network meta-analysis combining direct and indirect comparisons. ${ }^{3}$ Rifampicin-minocycline impregnated and heparin bonded catheters were most effective in reducing related bloodstream infection.

The question "effective for whom?" firstly needs to be turned on its head. Are there patients in whom impregnation of CVCs would act through such different physiological mechanisms that existing trial results do not apply? We argued this for young children when we undertook the CATCH trial (www.catchtrial. org.uk), a three arm trial of heparin bonded or rifampicin-minocycline $\mathrm{CVCs}$ versus standard CVCs in children in paediatric intensive care, because small lumens are associated with high rates of thrombosis, potentially over-riding antimicrobial impregnation.

Secondly, if results apply, do the absolute benefits outweigh the extra cost of impregnation? New guidelines recommend impregnated CVCs only in high risk patients or when effective programmes to reduce CVC infection do not exist. Yet a UK cost effectiveness study calculated that impregnated CVCs are cost effective even at bloodstream infection rates as low as $0.2 \%$, lower than rates seen in many intensive care units. ${ }^{4}$
Given this abundance of evidence, the puzzle is why the uptake of impregnated CVCs is limited and their use not supported in guidelines. What further evidence is needed? More head to head trials would give direct evidence on type of impregnation on bloodstream infection. We should be sceptical about the limited evidence for a lack of effect of impregnation on sepsis and all cause mortality because of low power and intensive treatment responses that will bias towards a null effect. And no more trials on catheter colonisation, a biased and clinically irrelevant outcome.

Competing interests: We are members of the CATCH Trial Team (NIHR HTA project number 08/13/47).

Full response at: www.bmj.com/content/347/bmj.f6570/rr/673640.

Smith RN, Nolan JP. Central venous catheters. BMJ 2013;347:66570. (11 November.)

2 Lai NM, Chaiyakunapruk N, Lai NA, O'Riordan E, Pau WSC, Saint S. Catheter impregnation, coating or bonding for reducing central venous catheter-related infections in adults. Cochrane Database Syst Rev 2013;6:CD007878.

3 Wang $\mathrm{H}$, Huang T, Jing J, Jin J, Wang P, Yang M, et al. Effectiveness of different central venous catheters for catheter-related infections: a network meta-analysis. $J$ Hosp Infect 2010;76:1-11.

4 Hockenhull J, Dwan K, Boland A, Smith G, Bagust A, Dündar Y, et al. The clinical effectiveness and cost-effectiveness of central venous catheters treated with anti-infective agents in preventing bloodstream infections: a systematic review and economic evaluation. Health Technol Assess Rep 2008;12:1-154.

5 Geffers C, Zuschneid I, Eckmanns T, Rüden H, Gastmeier P. The relationship between methodological trial quality and the effects of impregnated central venous catheters. Intens Care Med 2003;29:403-9.

Cite this as: BMJ 2013;347:f7169

๑ BMJ Publishing Group Ltd 2013 